\title{
Trauma-Induced Retroperitoneal Paraganglioma Rupture Which Was Misunderstood as a Massive Retroperitoneal Hemorrhage
}

\author{
Hongjin Shim ${ }^{1}$, Ji Young Jang ${ }^{1}$, Yeong Ju Kim², Jung Soo Lim ${ }^{3}$, and Keum Seok Bae ${ }^{1}$ \\ Departments of ${ }^{1}$ Surgery, ${ }^{2}$ Radiology, and ${ }^{3}$ Internal Medicine, Yonsei University Wonju College of Medicine, Wonju, Korea
}

Paraganglioma is a very rare tumor and it can be diagnosed with catecholamine hypersecretion which induce a paroxysmal hypertension [1-3]. When it is ruptured by traumatic injury and the patient has a hemodynamic instability due to bleeding, preoperative evaluation and medical therapy are not impossible in acute phase $[4,5]$. Sometimes even though the patient has hypovolemic shock, catecholamine can mask the symptom of hypotension which confuses a clinician to make an adequate decision. Unfortunately, there is no definitive guideline of treatment for traumatic ruptured paraganglioma. Nevertheless, paroxysmal hypertension during shock and enhanced mass give us a clue for suspect of paraganglioma rupture in this case; it is difficult to distinguish paraganglioma rupture from retroperitoneal hematoma because of paroxysmal hypertension and enhanced ruptured mass.
Received on April 1, 2017

Revised on July 4, 2017

Accepted on July 5, 2017

\section{Keum Seok Bae}

Department of Surgery, Yonsei University Wonju College of Medicine, 20 Ilsan-ro,

Wonju 26426, Korea

Tel: +82-33-741-0573

Fax: +82-33-741-0574

E-mail: bksgs@yonsei.ac.kr

*No potential conflict of interest relevant to this article was reported.

A 73-year-old female was admitted to trauma center after rolling down on the stairs which induced abdominal contusion. She was suffering from abdominal pain. Initial
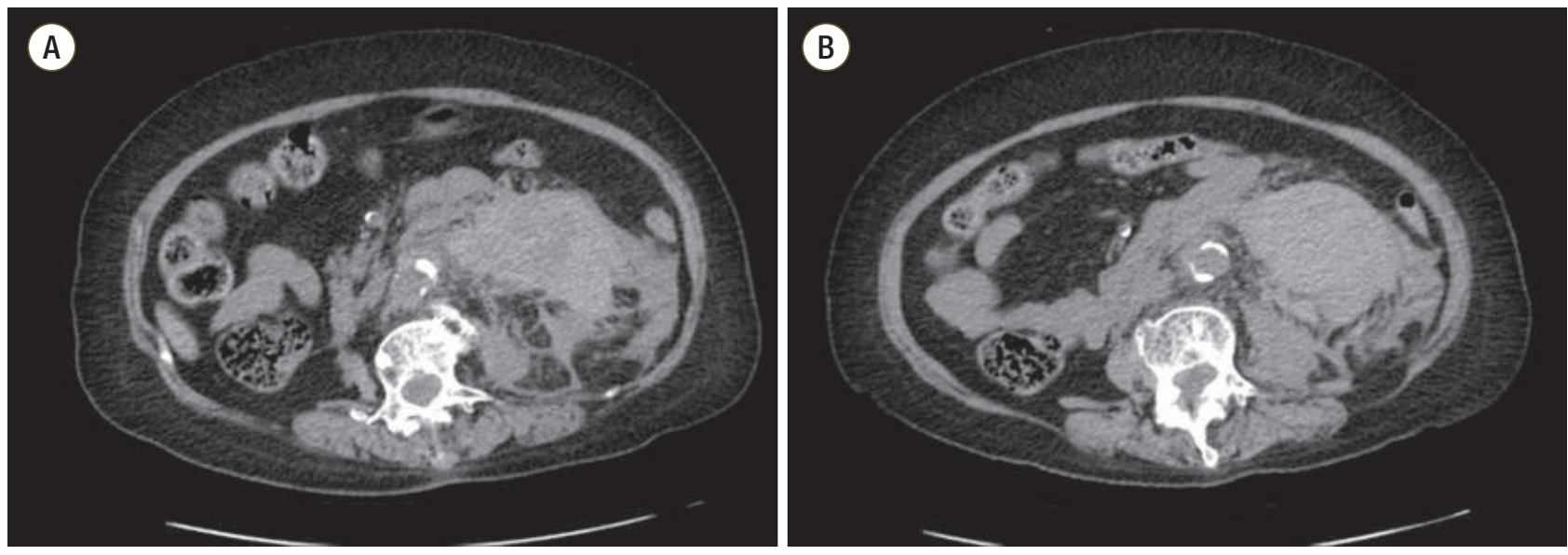

Figure 1. Precontrast views in the initial computed tomography. (A) Retroperitoneal hemorrhage was infiltrated in the left retroperitoneum. (B) A huge mass was not distinguished from the hematoma in the left retroperitoneum. 

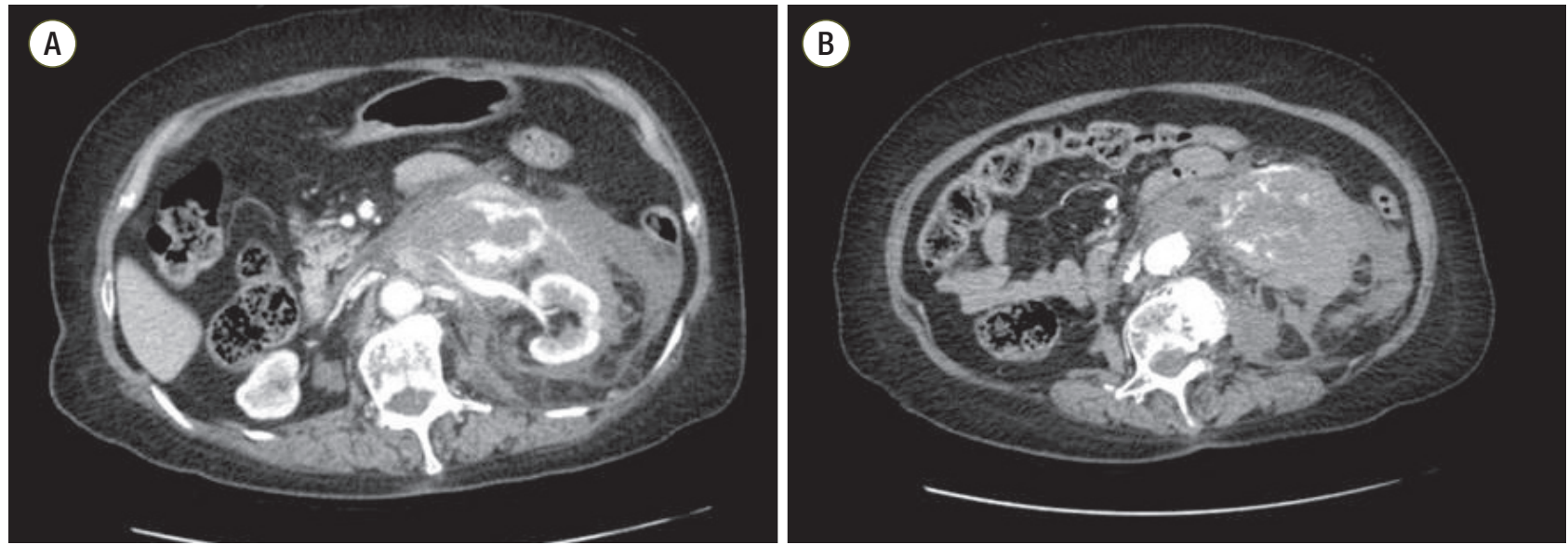

Figure 2. Postcontrast views in the initial abdominal computed tomography. (A) There were several extravasated lesions near the left kidney. (B) Some arteries were supplying to the paraganglioma.

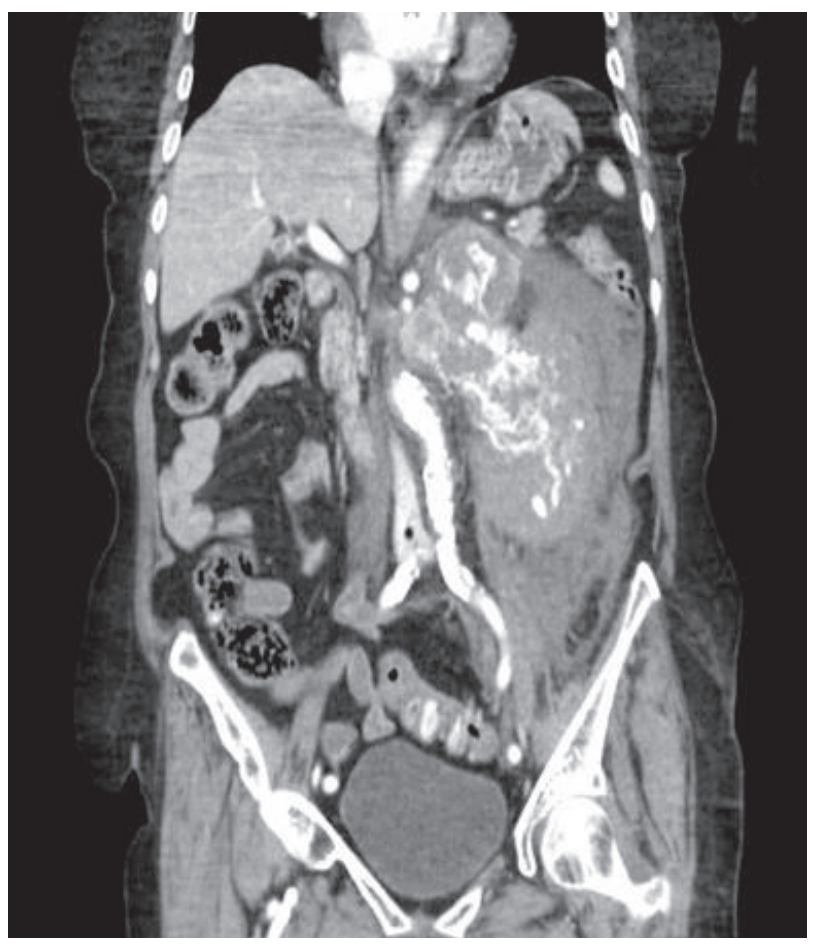

Figure 3. Postcontrast coronal view in the initial abdominal computed tomography: adjacent hemorrhage is masking the perirenal mass; therefore, it is difficult to distinguish from retroperitoneal hematoma.

blood pressure was $63 / 50 \mathrm{mmHg}$ and pulse rate was 46 / min. In an hour after resuscitation, blood pressure became too high $(241 / 114 \mathrm{mmHg})$ and fluctuated without any intervention. On the computed tomography scan, a huge mass with enhanced lesions with extravasation and adjacent hemorrhages were observed in the left retroperi-

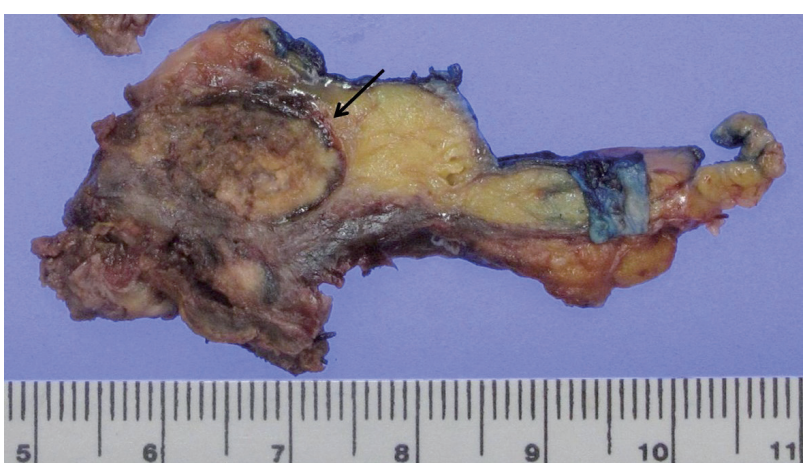

Figure 4. Gross finding of the paraganglioma. There is a lobulated brown solid mass with hemorrhage (arrow) in the soft tissue.

toneum (Figures 1-3). At the first time, emergency operation was considered to control bleeding but not performed because paroxysmal hypertension existed. The rupture of endocrine tumor secreting catecholamine was suspected. After laboratory evaluation with an endocrinologist and a radiologist, the mass turned out to be a paraganglioma.

After preoperative evaluation for paraganglioma, the medication for adrenal suppression was given to the patient for 14 days in the intensive care unit, then elective surgery was performed 1 month later. On the pathologic report, the specimen was identified as a paraganglioma with low risk of malignancy (pheochromocytoma of the Adrenal Gland Scaled Score 0) (Figures 4-6). 

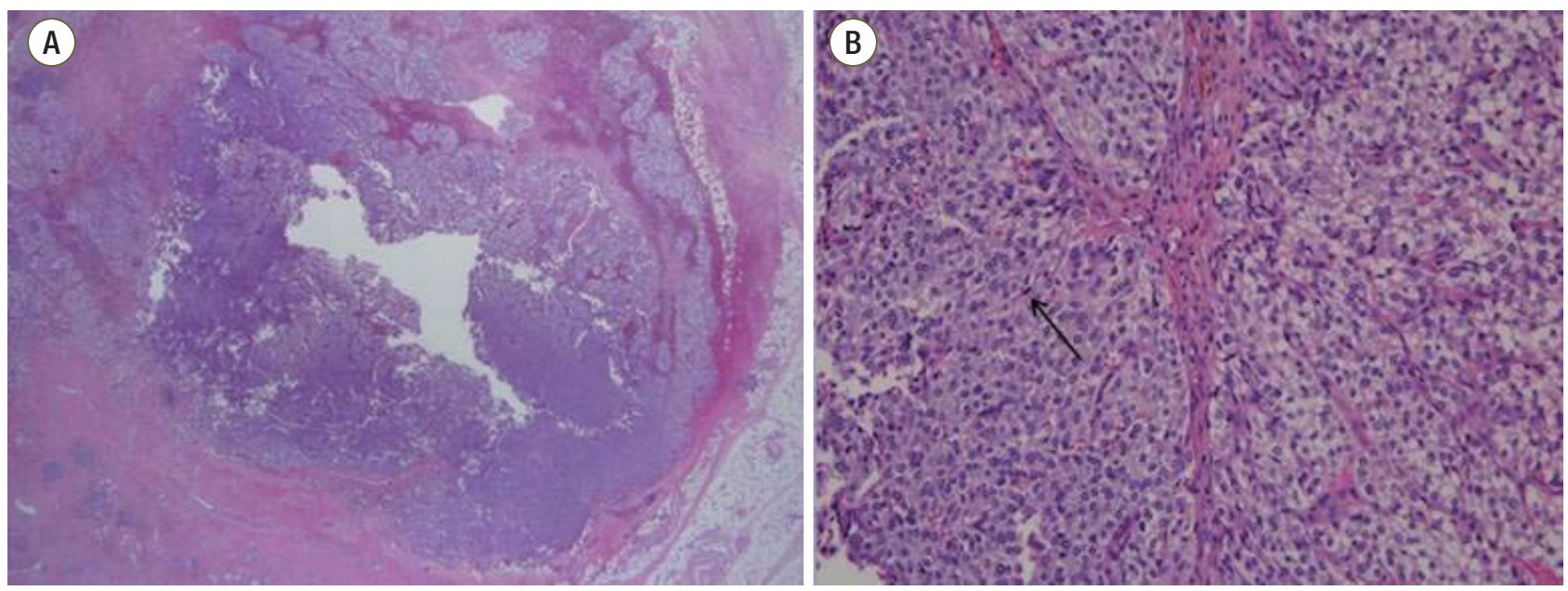

Figure 5. H\&E stain. (A) There is a lobulated solid mass with hemorrhage in the soft tissue $(\times 1.25)$. (B) Histology revealed an alveolar pattern with nest of neoplastic cells surrounded by vascularized connective-tissue septa $(\times 200)$. Nests outlined by sustentacular cells (arrow). Cells have finely granular basophilic or amphophilic cytoplasm. There was no necrosis.
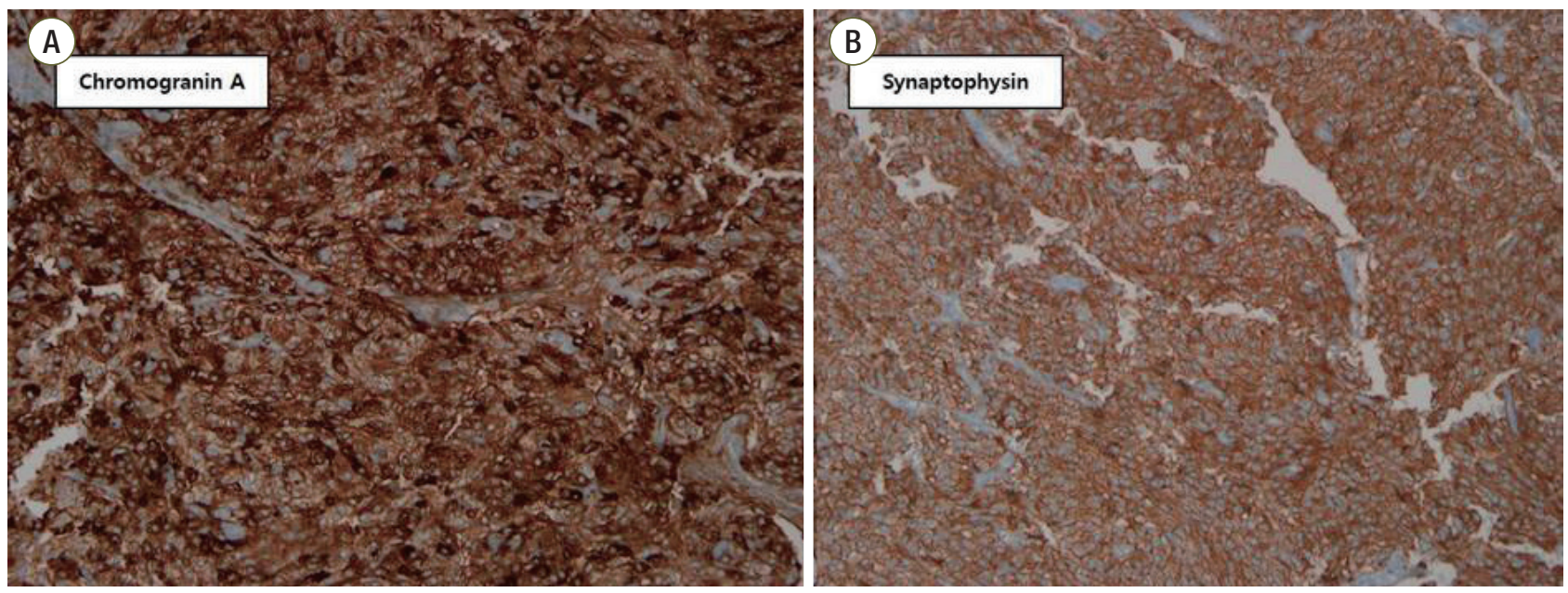

Figure 6. Immunohistochemical staining. Staining was positive for $(A)$ chromogranin A (×200) and (B) synaptophysin $(\times 200)$.

\section{ORCID}

Hongjin Shim

https://orcid.org/0000-0003-3073-3806

Ji Young Jang

https://orcid.org/0000-0001-6372-4194

\section{REFERENCES}

1. Pedroso C, Robalo R, Sereno P, Barros C, Marques C. A rare abdomino-pelvic tumor: paraganglioma. Acta Med Port 2015;28:114-6.

2. Pałasz P, Adamski Ł, Studniarek M. Paragangliomas: a propos of two cases. Diagnostics and treatment. Pol
J Radiol 2015;80:411-6.

3. Yau KK, Siu WT, Li MK. Unusual cause of acute abdomen: ruptured retroperitoneal paraganglioma. Asian J Surg 2008;31:32-5.

4. Mahmoudieh L, Saeedinia A, Ahmadpoor P, Temannaie Z, Parvin M, Torbati P, et al. Nephroquiz 8: perioperative management of paraganglioma. Iran J Kidney Dis 2015;9:259-62.

5. Sapienza P, Tedesco M, Graziano P, Moretti M, Mingazzini PL, Cavallaro A. An unusual case of spontaneous rupture of a clinically "silent" phaeochromocytoma. Anticancer Res 1997;17:717-20. 